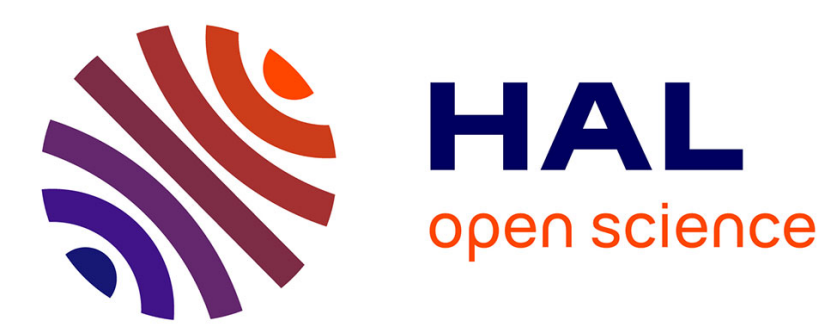

\title{
A SIMPLE MODEL FOR THE MOTION OF PULSED-LASER-DRIVEN THIN FLYERS
}

\author{
R. Lawrence, W. Trott
}

\section{To cite this version:}

R. Lawrence, W. Trott. A SIMPLE MODEL FOR THE MOTION OF PULSED-LASERDRIVEN THIN FLYERS. Journal de Physique IV Proceedings, 1991, 01 (C3), pp.C3-453-C3-458. 10.1051/jp4:1991364 . jpa-00250508

\section{HAL Id: jpa-00250508 https://hal.science/jpa-00250508}

Submitted on 1 Jan 1991

HAL is a multi-disciplinary open access archive for the deposit and dissemination of scientific research documents, whether they are published or not. The documents may come from teaching and research institutions in France or abroad, or from public or private research centers.
L'archive ouverte pluridisciplinaire HAL, est destinée au dépôt et à la diffusion de documents scientifiques de niveau recherche, publiés ou non, émanant des établissements d'enseignement et de recherche français ou étrangers, des laboratoires publics ou privés. 


\title{
A SIMPLE MODEL FOR THE MOTION OF PULSED-LASER-DRIVEN THIN FLYERS
}

\section{R.J. LAWRENCE and W.M. TROTT}

Sandia National Laboratories(1), P.O. Box 5800, Albuquerque, NM 87185, U.S.A.

\begin{abstract}
A model that predicts the final velocity of high-power, pulsed-1aserdriven thin flyers is described. The required input parameters can either be obtained from standard handbooks or simply extracted from one set of data. The model yields a number of features and scaling laws that are well verified by experiment. Specific comparisons of model predictions with experimental results illustrate excellent agreement for variations of laser fluence and pulse width as well as flyer diameter and thickness.
\end{abstract}

\section{Introduction}

Previous work [1] has shown that simple models can be used to describe the recoil momentum generated by the explosive vaporization caused by high-power pulsed radiation loads incident on target surfaces (e.g., from lasers). In most earlier investigations the vaporized blowoff products were allowed to expand freely. In the present study, short high-intensity laser pulses are transmitted through fused silica optical fibers onto thin-foil metallic flyers placed on the fiber ends. In this case the blowoff reactions are fully tamped by the fiber, and free expansion does not occur. The configuration is shown schematically in Figure 1 . To describe this situation, we have developed a simple model, employing conservation of energy and momentum, in the framework of the well-known Gurney theory for high-explosive-driven plates [2,3].

This analysis provides a good match to all major features of the relevant experimental measurements, including the threshold for flyer motion and the detailed dependence of the flyer velocity on energy fluence, pulse width, and flyer thickness. In addition to flyer velocity, the model predicts the energy coupling

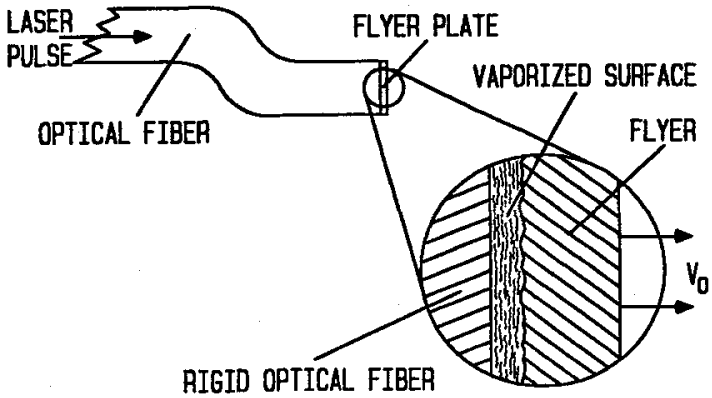

Fig 1. Schematic for laser-driven flyers.

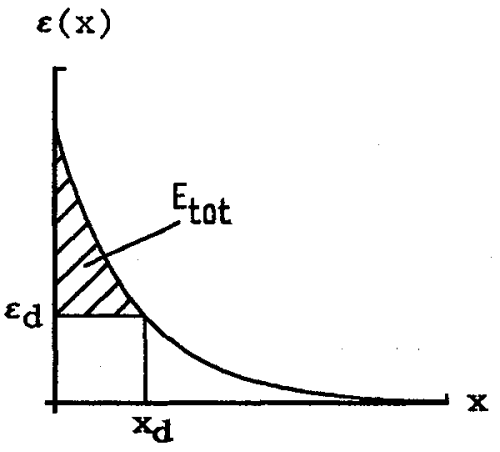

Fig 2. Energy deposition schematic.

Supported by the U.S. Department of Energy under contract No. DE-AC04-76DP00789. 
efficiency, the impulse (momentum) coupling coefficient, and the amount of flyer material removed in the blowoff process. At energy fluences well above the threshold, it is found, both theoretically and experimentally, that the velocity depends only very weakly on the laser pulse width, but scales with the square root of the fluence, inversely with the square root of the flyer thickness, and is inversely related to the overall effective energy loss. The success of this modeling approach is due to the fact that the late-time recoil momentum of the flyer is relatively insensitive to the details of the radiation interaction processes. Indeed, the latter is true for all interactions that depend principally on momentum conservation.

Most of the requisite material properties (e.g., density, decomposition energy, thermal diffusivity) can be obtained from standard compilations. The two exceptions are the integrated effective energy loss (due to reflection and radiation) and the effective absorption coefficient. The energy loss depends directly on the high-fluence coupling efficiency, which can be measured easily; and the effective absorption coefficient is then defined uniquely by the peak impulse coupling coefficient, which always occurs at some intermediate fluence. Thus both of these effective properties can be obtained from one set of experimental data. Extrapolation to other conditions is then straightforward. The process can be repeated for different flyer materials and laser wavelengths.

\section{Description of Model}

In developing this model, we make several simplifying assumptions. The most basic is that the deposition, vaporization, and driving processes are all one dimensional. This is equivalent to saying that the flyer is thin compared to its lateral dimensions. Unquestionably, some of the vaporized blowoff products escape around the periphery of the flyer in a two-dimensional fashion, but this seems to occur at relatively late times, after the acceleration is largely complete. With regard to the actual physical processes that occur, we assume that the end of the optical fiber is transparent to the incident laser radiation, but mechanically rigid with respect to the blowoff. While the blowoff products are accelerating the flyer, we further assume that their spatial velocity profile is linear, from zero at the rigid fiber end, to the flyer velocity $v_{0}$ at the blowoff-solid interface. This is the basic approximation contained in the Gurney theory, which we are employing in the present model. Finally, we assume that after being deposited exponentially in the flyer, as suggested in Figure 2, the energy in the blowoff material equilibrates over the specified deposition time. This is related both to the Gurney approximation and to the insensitivity of the delivered impulse to the details of the deposition and interaction phenomena.

In the present situation we apply the Gurney theory by equating the "potential" energy deposited in the flyer with the kinetic energy of both the blowoff products and the remaining solid portion of the plate. Note that in this one-dimensional approximation the energies are per unit area. The result is,

$$
\rho \mathrm{x}_{\mathrm{d}} \mathrm{E}=(\rho / 2)\left(\mathrm{x}_{0}-\mathrm{x}_{\mathrm{d}}\right) \mathrm{v}_{\mathrm{o}}^{2}+(\rho / 2) \int_{0}^{\mathrm{x}_{\mathrm{d}}}\left(\mathrm{v}_{\mathrm{o}} \mathrm{x} / \mathrm{x}_{\mathrm{d}}\right)^{2} \mathrm{dx} .
$$

The left hand side represents the energy available for accelerating the flyer, where $E$ is the so-called Gurney energy, $\rho$ is the flyer density, and $x_{d}$ is the thickness of the flyer that is vaporized by the laser pulse. This thin blowoff layer is analogous to the high explosive in the original form of the Gurney theory. The first term on the right is the kinetic energy of the flyer, which has an original thickness $\mathrm{x}_{0}$, and a final uniform velocity $\mathrm{v}_{0}$. The remaining term on the right is the kinetic energy of the blowoff material, where we have assumed a Lagrangian velocity profile of the form

$$
\mathrm{v}(\mathrm{x})=\left(\mathrm{v}_{\mathrm{o}} / \mathrm{x}_{\mathrm{d}}\right) \mathrm{x}, \quad 0 \leq \mathrm{x} \leq \mathrm{x}_{\mathrm{d}},
$$

as described above. We can solve Eq (1) for the flyer velocity, obtaining 


$$
\mathrm{v}_{\mathrm{o}}=\left[\frac{3 \mathrm{E}}{3 \mathrm{x}_{\mathrm{o}} / 2 \mathrm{x}_{\mathrm{d}}-1}\right]^{1 / 2} .
$$

This equation presents no difficulties unless $x_{d} \geq 3 x_{0} / 2$, a situation that can never occur.

To define the Gurney energy. $E$, we first assume that the laser energy is deposited exponentially in the flyer as sketched in Figure 2. The energy per unit mass $\varepsilon(x)$ is then

$$
\varepsilon(\mathrm{x})=\mu_{\text {eff }} F_{\circ}(1-\mathrm{r}) e^{-\mu_{\text {eff }} \rho \mathrm{x}},
$$

where $F_{O}$ is the incident laser fluence, $r$ is the effective energy loss (combining both reflective and radiative losses), and $\mu_{\text {eff }}$ is the effective absorption coefficient. If the flyer material has a decomposition or vaporization energy $\varepsilon_{d}$, then Eq (3) can be solved for the thickness of the blowoff layer $x_{d}$ by setting $\varepsilon\left(x_{d}\right)=\varepsilon_{d}$, resulting in

$$
\mathrm{x}_{\mathrm{d}}=\frac{1}{\mu_{\text {eff }} \rho} \ln \frac{\mu_{\mathrm{eff}} \mathrm{F}_{\mathrm{o}}(1-\mathrm{r})}{\varepsilon_{\mathrm{d}}} .
$$

Now the amount of energy available for conversion to kinetic energy is that in excess of $\varepsilon_{d}$ and contained in the blowoff layer, as indicated by $E_{\text {tot }}$ in Figure 2. It is

$$
E_{\text {tot }}=\int_{0}^{x_{d}}\left[\varepsilon(x)-\varepsilon_{d}\right] d x,
$$

which, with Eqs (3) and (4), can be integrated to yield

$$
E_{\text {tot }}=\frac{F_{o}(1-r)}{\rho}-\frac{\varepsilon_{\mathrm{d}}}{\mu_{\text {eff }} \rho}\left(1+\ln \frac{\mu_{\text {eff }} F_{o}(1-r)}{\varepsilon_{d}}\right) .
$$

The Gurney energy is then taken to be this value as equilibrated over the blowoff material,

$$
E=E_{\text {tot }} / x_{d}
$$

It remains to define the effective absorption coefficient $\mu_{\text {eff }}$. We take

$$
\mu_{\text {eff }}=\frac{\mu_{\mathrm{a}}}{1+\mathrm{k} \mu_{\mathrm{a}} \rho(\alpha \tau)^{\frac{1 / 2}{2}}},
$$

where $\mu_{\mathrm{a}}$ is the true absorption coefficient, $\alpha$ is the thermal diffusivity, and $\tau$ is the laser pulse width. The constant $k$ allows for uncertainties in handbook values for $\mu_{\mathrm{a}}$ and $\alpha$, and thus permits extrapolation of the solution to other pulse widths; it is not an additional material parameter. In the limit of short pulse widths $\mu_{\text {eff }}$ reduces to the true absorption coefficient $\mu_{a}$, with a mean free path of $1 / \mu_{\mathrm{a}} \rho$. For long pulse widths, such that $\tau \gg 1 / \alpha\left(\mathrm{k} \mu_{\mathrm{a}} \rho\right)^{2}$, the problem is thermally dominated, and the effective mean free path becomes $k(\alpha \tau)^{\frac{1 / 2}{2}}$, which is a characteristic diffusion length for a pulse width $\tau$.

The complete model for predicting the flyer velocity is implemented by first

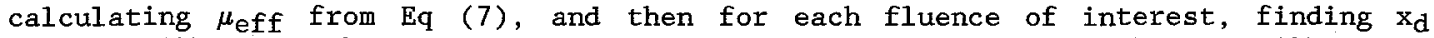
from Eq (4), E tot from Eq (5), E from Eq (6), and finally, $v_{0}$ from Eq (2). Two additional quantities of interest include the coupling efficiency $f$, which is 
defined as the ratio of the flyer kinetic energy to the input energy fluence, and is given by

$$
f=\frac{\rho\left(\mathrm{x}_{\mathrm{o}}-\mathrm{x}_{\mathrm{d}}\right) \mathrm{v}_{\mathrm{o}}^{2}}{2 \mathrm{~F}_{\mathrm{o}}},
$$

and the impulse coupling coefficient $\mathrm{I} / \mathrm{F}_{\mathrm{O}}$,

$$
I / F_{0}=\frac{\rho\left(x_{0}-x_{d}\right) v_{0}}{F_{0}} .
$$

The latter is defined as the momentum in the flyer divided by the energy in the laser pulse.

Features of the Mode1

This formulation leads to a number of features that describe how these laserdriven flyers operate. First, there is a threshold fluence $F_{\text {th }}$ below which neither vaporization nor flyer motion can occur. Its value can be determined by setting $\mathrm{x}_{\mathrm{d}}=0$ in $\mathrm{Eq}(4)$, leading to

$$
F_{t h}=\frac{\varepsilon_{d}}{\mu_{\text {eff }}(1-r)} .
$$

If we assume that $x_{d} \ll x_{0}$, then at high fluences, where $F_{0} \gg F_{t h}$, we can show from Eqs (2), (6), and (5) that the flyer velocity behaves as

$$
\mathrm{v}_{\mathrm{o}} \approx\left[\frac{2 \mathrm{~F}_{\mathrm{o}}(1-\mathrm{r})}{\rho \mathrm{x}_{\mathrm{O}}}\right]^{1 / 2},
$$

and is thus independent of the pulse width $\tau$, which appears only in $\mu_{\mathrm{eff}}$. In a similar fashion we can show from Eq (8) that the coupling efficiency $f$ becomes

$$
f \approx(1-r), \quad x_{d} \ll x_{0} \text { and } \quad F_{0} \gg F_{t h} .
$$

This last equation is important because it allows us to determine the effective energy loss $r$ directly from an experimental measurement of the high-fluence asymptotic value of $f$, which is independent of everything but $r$.

Also of importance is the observation that the impulse coupling coefficient $\mathrm{I} / \mathrm{F}_{\mathrm{O}}$ has a peak value, which with $\mathrm{x}_{\mathrm{d}} \ll \mathrm{x}_{\mathrm{O}}$ and $\mathrm{Eq}(9)$, can be shown to be

$$
\left(I / F_{0}\right)_{\max } \approx 0.45(1-r)\left(\rho x_{0} \mu_{e f f} / \varepsilon_{d}\right)^{\frac{1}{2}} \text {, }
$$

at a fluence of $3.51 \mathrm{~F}_{\mathrm{th}}$. Once $r$ has been determined from Eq (12), an experimental estimate for $\left(I / F_{0}\right)_{\max }$, along with $\mathrm{Eq}(13)$, leads to a value for $\mu_{\text {eff }}$. These two constants, $\mathrm{r}$ and $\mu_{\text {eff }}$, are the only parameters not readily obtained from standard compilations.

\section{Comparison With Experiment}

Experimental results on aluminum flyers using a configuration similar to that shown in Figure 1 have been reported by Trott and Meeks [4]. Since that work was completed, a considerable body of additional data has been obtained. In Figure 3 we show three data sets in which the laser pulse width (at $\lambda=1.06 \mu \mathrm{m}$ ) and flyer thickness were held constant. After the available material properties were obtained from standard references, the points labeled "nominal" were used to determine $r$ and $\mu_{\text {eff }}$ as outlined in conjunction with Eqs (12) and (13). Knowing the experimental pulse width and the other parameters, the absorption coefficient constant $k$ can be 


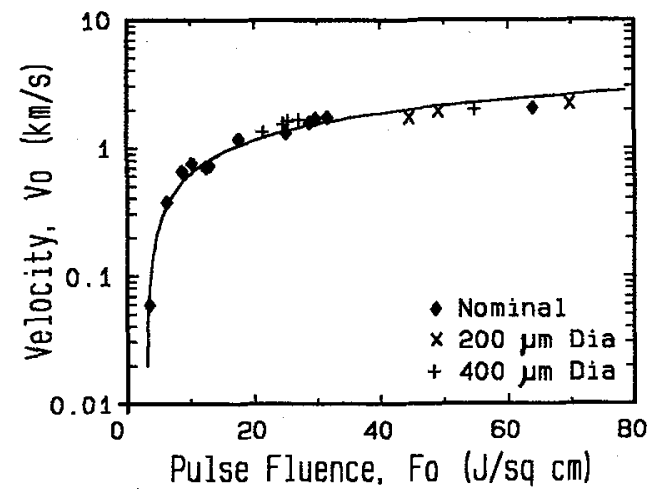

Fi.g 3. Flyer velocity as function of fluence for $\mathrm{x}_{\mathrm{O}}=25 \mu \mathrm{m}$ and $\tau=25 \mathrm{~ns}$.
Table I

MATERIAL PROPERTIES FOR ALUMINUM

Material Density, $\rho$

$\left(\mathrm{g} / \mathrm{cm}^{3}\right)$

Decomposition Energy, $\varepsilon_{\mathrm{d}}$ $(\mathrm{kJ} / \mathrm{g})$

Thermal Diffusivity, $\alpha$ $\left(\mathrm{cm}^{2} / \mathrm{s}\right)$

True Absorption Coefficient, $\mu_{\mathrm{a}}$ $\left(\mathrm{cm}^{2} / \mathrm{g}\right)$ $4.4 \times 10^{5}$

Absorption Coefficient Constant, $k$ (Dimensionless)

0.2533

Effective Energy Loss, r

(Dimensionless)

calculated with the aid of $\mathrm{Eq}$ (7). The resultant set of material parameters required by the model is shown in Table $I$. The three sets of data in Figure 3 were taken on different occasions and with two different flyer diameters (the nominal data also employed $400 \mu \mathrm{m}$ optical fibers). The facts that they are all consistent and all agree well with the model calculations, indicated by the solid line, show that the system is indeed, for all practical purposes, one dimensional. It is also evident that for high fluences the flyer velocity scales with the square root of the fluence, as suggested by $\mathrm{Eq}$ (11).

The first major departure from the conditions of Figure 3 was an attempt to throw flyers that were a factor of five thinner than the nominal. The data, as shown in Figure 4, were all taken with an initial flyer thickness of $\mathrm{x}_{0}=5 \mu \mathrm{m}$ and a laser pulse width of $\tau \approx 18 \mathrm{~ns}$. Other than changing $\mathrm{x}_{0}$ and $r$, the model calculations indicated by the solid line use exactly the same parameters as were employed for Figure 3. By comparing flyer velocities at constant fluences that are well above the knee of the curve, we see that the flyer-thickness scaling predicted by Eq (11) seems to hold. That is, $v_{0} \sim x_{0}^{-\frac{1}{2}}$ for $F_{0} \gg F_{t h}$.

The other principal experimental parameter to be varied was the laser pulse width, which was reduced by a factor of five from the conditions of Figure 3 . Although there is some scatter, most of the experiments were run with $4 \leq \tau$ $\leq 5$ ns. In this case the flyer thickness was $x_{0}=12.7 \mu \mathrm{m}$. The results are shown, along with the theoretical predictions, in Figure 5. Again, the model calculations were performed with changes in $x_{0}$ and $\tau$ only. We find that the differences in the high-fluence flyer velocity between these conditions and those represented by the other figures can be explained by the observed and predicted flyer-thickness scaling alone. Thus, as was anticipated, this velocity is

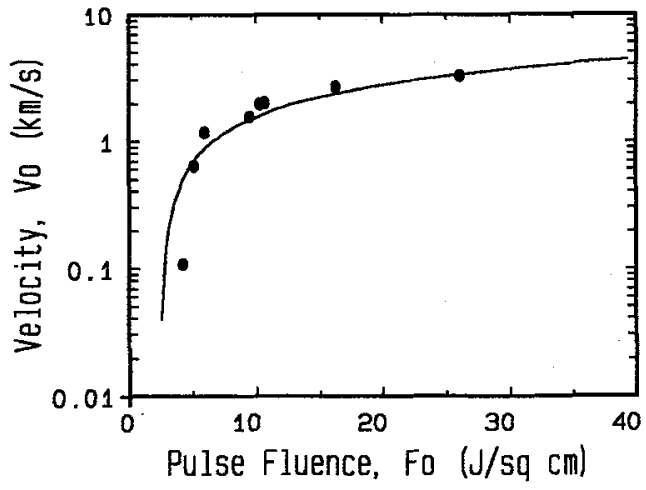

Fig 4. Flyer velocity as function of fluence for $x_{0}=5 \mu \mathrm{m}$ and $\tau=18 \mathrm{~ns}$.

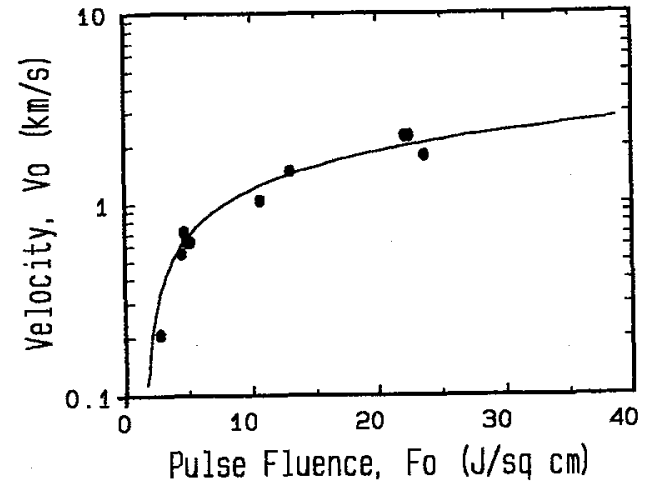

Fig 5. Flyer velocity for $\mathrm{x}_{0}=12.7 \mu \mathrm{m}$ and $\tau=4$ to $5 \mathrm{~ns}$. 
essentially independent of the pulse width $\tau$. This is an important result because it suggests that as long as the system is operated with $F_{0} \gg F_{t h}$ a desired flyer velocity can be achieved almost without regard to the laser pulse width.

For lower fluences, a careful examination of the figures shows that the threshold fluence scales with the square root of the pulse width. This would be expected if we were operating in the thermally dominated regime. We can show this by substituting Eq (7) into Eq (10) with the assumption of large $r$, obtaining,

$$
F_{\mathrm{th}} \approx \frac{\mathrm{k} \rho \varepsilon_{\mathrm{d}}(\alpha \tau)^{\frac{1}{2}}}{1-\mathrm{r}}, \quad \tau \gg 1 / \alpha\left(k \mu_{\mathrm{a}} \rho\right)^{2} .
$$

The fact that this scaling applies leads to the conclusion that even with pulse widths as short as 4 ns, thermal diffusion controls the energy deposition

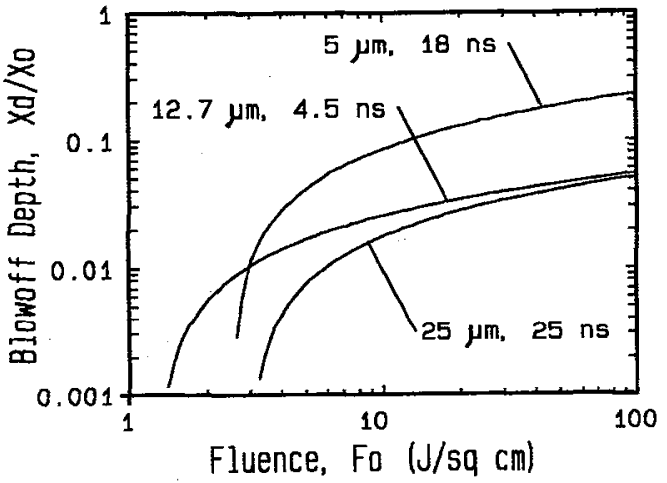

Fig 6. Blowoff depth for the three different experimental conditions. process, and the true absorption coefficient $\mu_{a}$ is relatively unimportant in the processes occurring here.

As a final point we compare the thicknesses of the blowoff layer for each of the experimental situations under consideration. Since we cannot currently verify these results, we show only the model predictions in Figure 6. The curves are labeled with the flyer thickness $x_{0}$ and pulse width $\tau$ respectively. We obtain the largest fractional blowoff thickness for the conditions of Figure 4. In this case

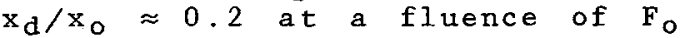
$\approx 26 \mathrm{~J} / \mathrm{cm}^{2}$. For virtually all of the other conditions examined we find that $x_{d} / x_{0}<0.1$. This should be adequate to fulfill the requirements of the "thick-flyer" assumption $\left(\mathrm{x}_{\mathrm{d}}<\mathrm{x}_{\mathrm{o}}\right.$ ) that we made for several of our derivations.

Closure

We have developed a simple model for predicting the late-time velocity of thin flyers driven by high-power pulsed radiation loads under conditions where the blowoff products are fully tamped. Once the full set of required parameters are established by one set of data, we have found the model capable of accurate extrapolation to other conditions involving changes in energy fluence, laser pulse width, and flyer thickness and diameter. Noting that we have examined only one material, aluminum, our plans for the near future call for a study of other flyer materials in an attempt to find alternates that may be more efficient in converting laser energy into flyer kinetic energy. This would improve the potential for these types of fiber-optic systems in a number of different areas of application.

References

1/ LAWRENCE, R. J., An Effective Properties Model for Pulsed Radiation Interactions, SAND 88-0245, Sandia National Laboratories, Albuquerque, NM (1988).

/2/ GURNEY, R. W., The Initial Velocities of Fragments From Bombs, She11, and Grenades, BRL Report No. 405, Ballistic Research Laboratories, Aberdeen Proving Ground, MD (1943).

/3/ KENNEDY, J. E., Gurney Energy of Explosives: Estimation of the Velocity and Impulse Imparted to Driven Metal, SC-RR-70-790, Sandia Laboratories, Albuquerque, NM (1970).

14. TROTT, W. M. and K. D. MEEKS, "Acceleration of Thin Foil Targets Using FiberCoupled Optical Pulses," in Shock Compression of Condensed Matter - 1989, S. C. Schmidt et al, Eds., Elsevier Science Publishers B.V. (1990). 